Revue musicale OICRM

\title{
Le Courrier musical et le premier conflit mondial (1904-1923). Propagande, mobilisation culturelle et sortie de guerre
}

\section{Martin Guerpin}

Volume 4, numéro 2, 2017

Enjeux culturels dans la presse musicale française, 1900-1925

URI : https://id.erudit.org/iderudit/1043219ar

DOI : https://doi.org/10.7202/1043219ar

Aller au sommaire du numéro

\section{Éditeur(s)}

Observatoire interdisciplinaire de création et recherche en musique (OICRM)

ISSN

2368-7061 (numérique)

Découvrir la revue

Citer cet article

Guerpin, M. (2017). Le Courrier musical et le premier conflit mondial (1904-1923). Propagande, mobilisation culturelle et sortie de guerre. Revue musicale OICRM, 4(2), 35-57. https://doi.org/10.7202/1043219ar
Résumé de l'article

Cet article étudie et remet en perspective le rôle joué par Le Courrier musical dans la mobilisation culturelle du monde musical français pendant la Grande Guerre. Il montre tout d'abord que, de 1916 à 1918, la revue sert d'organe de diffusion d'un discours de propagande nationaliste et antigermanique. Une comparaison de ce discours avec les articles publiés avant et après le conflit permet ensuite de nuancer l'idée selon laquelle la guerre constitua une période d'exception dans l'histoire du positionnement esthétique et idéologique de la revue. Elle marqua bien plutôt une reconfiguration de discours nationalistes très présents dans Le Courrier musical dès les années 1900. D'autre part, ces discours perdurent plusieurs années après 1918. Dans la lignée des travaux récents sur les entrées et sorties de guerre, cet article entend montrer que la compréhension de l'histoire de la musique pendant les conflits implique de ne pas se cantonner à la chronologie traditionnelle et officielle des déclarations de guerre et des traités de paix. 


\title{
Le Courrier musical et le premier conflit mondial (1904-1923). Propagande, mobilisation culturelle et sortie de guerre
}

\author{
Martin Guerpin
}

\begin{abstract}
Résumé
Cet article étudie et remet en perspective le rôle joué par Le Courrier musical dans la mobilisation culturelle du monde musical français pendant la Grande Guerre. Il montre tout d'abord que, de 1916 à 1918, la revue sert d'organe de diffusion d'un discours de propagande nationaliste et antigermanique. Une comparaison de ce discours avec les articles publiés avant et après le conflit permet ensuite de nuancer l'idée selon laquelle la guerre constitua une période d'exception dans l'histoire du positionnement esthétique et idéologique de la revue. Elle marqua bien plutôt une reconfiguration de discours nationalistes très présents dans Le Courrier musical dès les années 1900. D'autre part, ces discours perdurent plusieurs années après 1918. Dans la lignée des travaux récents sur les entrées et sorties de guerre, cet article entend montrer que la compréhension de l'histoire de la musique pendant les conflits implique de ne pas se cantonner à la chronologie traditionnelle et officielle des déclarations de guerre et des traités de paix.
\end{abstract}

Mots clés : critique musicale ; guerre ; musique ; presse ; propagande.

\begin{abstract}
This article explores the role of the Courrier Musical in the cultural mobilization of the French musical world during the Great War. It shows how, from 1916 to 1918, the journal played a crucial role in spreading a nationalist and anti-German propaganda. A comparison between this propaganda and articles published before and after the war then leads to qualify the idea that the war years were a singular and exceptional period in the history of discourses published in the Courrier musical. In fact, the war only brought to the foreground ideas and claims that had been regularly featured in the journal since the 1900s. Besides, Le Courrier musical also perpetuated these ideas and claims well after the official end of the Great War. Following recent historical studies on periods preceding and following the war, this article make a case for an understanding of the history of music during conflicts that does not restrict itself to the official dates of declarations of war and peace treatises.
\end{abstract}

Keywords: music; music criticism; press; propaganda; war. 
Depuis la fin des années 1990, 1'histoire de la musique pendant la Première Guerre mondiale a été envisagée du point de vue des répertoires, des sociétés de concerts, de l'édition musicale, ou encore des représentations de l'ennemi (Duchesneau 1996, Caballero 1999, Huybrechts 1999, Leterrier 2000, Watkins 2003, Buch 2004, AudoinRouzeau et al. 2009, Anselmini 2013, Doé de Maindreville et Etcharry 2014). Les travaux cités ont permis d'éclairer une période que les historiens de la musique tendaient auparavant à occulter, passant directement de la fin de la Belle Époque au début des Années folles. Mais paradoxalement, ces mêmes travaux reconduisent en creux le cloisonnement entre périodes de guerre et de paix : pour des raisons de découpage chronologique évidentes, les années étudiées sont celles du conflit, envisagé comme une période exceptionnelle et déconnectée de ce qui la précède et de ce qui la suit directement.

Or, de récentes études menées dans le domaine de l'histoire culturelle ont mis en évidence l'absence de coïncidence stricte entre l'émergence et la disparition des cultures de guerre et les bornes chronologiques officielles des conflits (déterminées par la signature des déclarations de guerre, des armistices et des traités de paix). À travers les notions d'entrées et de sorties de guerre(Becker 1977, Horne 2002, Cabanes et Piketty 2007, Baty-Delalande et Trévisan 2016), il a été montré que les phénomènes de mobilisation culturelle observables lors des conflits sont déjà à l'œuvre, à des degrés d'intensité certes moindres, avant la déclaration de guerre officielle. De même, la mobilisation peut se prolonger plusieurs années après la cessation des combats. Dans cet article, nous faisons l'hypothèse que ces phénomènes d'entrée et de sortie de guerre sont également à l'œuvre dans le domaine de la musique. Le Courrier musical (1899-1935) constitue un excellent cas d'étude pour tester cette hypothèse. D'abord intitulée Le Courrier musical, artistique et littéraire du littoral, la revue siège dans un premier temps à Menton et sert de bulletin d'actualité musicale aux hivernants de la Côte d'Azur. En 1900, Albert Diot en prend la direction. De concert avec René Doire, qu'il nomme rédacteur en chef en 1903 avant d'en faire son successeur en 1910, Diot fait subir à la revue une mue spectaculaire. Elle adopte ainsi son titre définitif et se dote d'une section qui regroupe des articles de fonds, consacrés à des analyses d'œuvres et des études d'esthétique où la question liée aux identités nationales occupe une place importante. Grâce à de prestigieux collaborateurs, Henry Gauthier-Villars, Lionel de La Laurencie, Camille Mauclair, Paul de Stœcklin Jean d'Udine, puis dans les années 1910, Michel-Dimitri Calvocoressi, Gaston Carraud ou encore Camille Saint-Saëns, Le Courrier musical s'impose comme l'une des revues musicales françaises les plus importantes et les plus diffusées de la Belle Époque. Elle le demeurera dans l'entre-deux-guerres.

Deux raisons en font une source privilégiée. D'une part, Le Courriermusical constitue un point d'observation idéal pour comprendre le rôle de la presse spécialisée, de la musicographie et de la critique musicale dans la mobilisation de la musique pendant la Première Guerre mondiale. Son étude complète ainsi les travaux menés jusqu'à aujourd'hui sur le rôle joué dans la culture de guerre par les musiciens, les répertoires sacrés et de circonstance, ainsi que les concerts et l'édition musicale (Becker 2000, Leterrier 2000, Anselmini 2013, Moore 2014). Avec La Musique pendant la guerre (1915-1917), étudiée dans ce numéro par Liouba Bouscant, elle est en effet l'une 
des rares publications spécialisées qui paraît pendant la Première Guerre mondiale. La Gazette des classes du Conservatoire (1914-1918) complète ce panorama. Toutefois, ce bulletin s'intéresse moins à l'actualité musicale qu'au maintien de la communauté musicienne des élèves et anciens élèves du Conservatoire (Segond-Genovesi 2009, p. 175-190). Les principales revues musicales de la Belle-Époque, Le Ménestrel, Le Monde musical, Le Guide du concert, ainsi que Comoedia, cessent de publier entre septembre 1914 et octobre 1919. Après deux ans d'interruption causés par l'éclatement du conflit, Le Courrier musical reparaît à partir du mois de décembre 1916. Dans l'éditorial de ce numéro, son directeur, René Doire, la présente de manière explicite comme un « ardent organe de propagande française » (Doire 1916, p. 1) qui doit contribuer à créer et maintenir l'Union sacrée du monde musical français afin de "gagner l'univers au génie artistique de notre race [française] "(ibid.). $\mathrm{Au}$ début $\mathrm{du} \mathrm{XX}^{\mathrm{e}}$ siècle, le terme " propagande " est ambivalent. Il n'est pas encore systématiquement chargé, comme c'est le cas aujourd'hui, de connotations négatives, pas plus qu'il n'est associé à des régimes non démocratiques (D'Almeida 2002). Il renvoie avant tout à la propagation sur un territoire national et à l'étranger d'informations et d'idées, sans nécessairement fair référence à des pratiques de désinformation et de manipulation de masse. C'est toutefois pendant la Première Guerre mondiale que le mot " propagande » commence à désigner une volonté de discréditer un ennemi, tant sur le plan politique que culturel (ibid., p. 143-144). L'éditorial de Doire manifeste très bien cette ambivalence : en ces temps de guerre, il entend faire de sa revue un organe de promotion de la musique française, tout en la mettant au service de l'effort de guerre. Ce faisant, Doire inscrit Le Courrier musical dans une logique de guerre totale, qui implique autant les soldats mobilisés que 1'arrière ${ }^{1}$ : "La France ne saurait vaincre sans la coordination parfaite, sans le concours intégral de tous ses éléments vitaux, de ceux-là même qui paraissent le plus étrangers à toute activité guerrière " (Doire 1916, p. 1). De ce point de vue, Le Courrier musical fait front commun avec La Musique pendant la guerre et la Gazette des classes du Conservatoire.

D'autre part, les textes publiés dans Le Courrier musical en amont et en aval du conflit font de la revue une source particulière et originale. Sa longévité avant et après la Première Guerre mondiale la distingue de ses deux consœurs. Elle en fait un point d'observation permettant d'observer la genèse et le devenir du discours de guerre, de part et d'autre de ses bornes chronologiques. De fait, ces textes reflètent un double débordement du discours de mobilisation culturelle développé de 1916 à 1918 : ce discours, à l'image des prend appui sur des thèmes développés dans les colonnes de la revue pendant les années 1900 et 1910. De même, une partie de ce discours y perdure plusieurs années après la fin de la guerre.

1 L'expression a été définie dès 1918, sous la plume de Léon Daudet : " Qu'est-ce que la guerre totale? C'est l'extension de la lutte, dans ses phases aigües comme dans ses phases chroniques, aux domaines politique, économique, commercial, industriel, intellectuel, juridique et financier. Ce ne sont pas seulement les armées qui se battent, ce sont aussi les traditions, les coutumes, les codes, les esprits, et surtout les banques » (Daudet 1918, p. 8). 
L'étude des articles publiés dans cette revue de la décennie 1900 à la décennie 1920 permettra, d'une part, de compléter les travaux sur la mobilisation du monde musical pendant la Guerre et, d'autre part, de mettre en évidence les traces de cette mobilisation avant et après le conflit. Trois questions nous guideront : en quoi les positions qui y sont défendues entre 1916 et 1918 rejoignent-elles les discours de propagande antiallemande ? L'affirmation de ces positions marque-t-elle une rupture dans l'histoire de la revue ou bien une radicalisation de discours déjà en circulation avant la guerre? Qu'advient-il, enfin, du discours de mobilisation culturelle après la fin des hostilités ?

\section{LA MOBILISATION DU COURRIER MUSICAL PENDANT LA GUERRE}

\section{Un instrument de propagande dans un contexte de guerre totale}

Dans Le Courrier musical, l'œuvre de propagande réclamée par Doire se traduit tout d'abord par d'incessants appels à l'Union sacrée du monde musical français. Conformément à cette Union, prônée par Raymond Poincaré dans son discours du 4 août 1914, les débats qui opposent différentes tendances esthétiques ou différentes institutions doivent passer au second plan derrière la nécessité de faire front commun face à l'Allemagne : "la France [...], explique Poincaré dans son message, sera héroïquement défendue par tous ses fils » (voir à ce sujet J.-J. Becker et A. Becker 1988, p. 22-43 et Audoin-Rouzeau et Becker 1995, p. 264-283). L'expression fait florès dans le domaine de la musique. En avril 1917, Charles Tenroc appelle de ses vœux une "Union sacrée des éditeurs » et la création d'une «Édition française de la Musique classique » (Tenroc 1917b) afin de se débarrasser de la concurrence de l'édition musicale allemande. La nécessité de l'Union sacrée motive également la création, le 10 mars 1916, de la Ligue nationale pour la défense de la musique française qui réunit plusieurs figures importantes du monde musical français : Camille Saint-Saëns, Vincent d'Indy, Gustave Charpentier, Théodore Dubois, Xavier Leroux ou encore Charles Lecocq. L'Union sacrée est encore mobilisée la même année pour justifier le projet de réunion de la Société nationale de musique (SNM) et la Société musicale indépendante (SMI) dans une Société nationale réunifiée (voir à ce sujet Duchesneau 1996). C'est au nom de cette Union que Gabriel Fauré plaide en faveur de la fusion. C'est en son nom encore que le compositeur Alfred Bertelin, l'un des collaborateurs de la revue les plus actifs pendant la guerre avec Doire et Tenroc, reproche à Maurice Ravel de la refuser (Fauré 1917, p. 133) :

En toute sincérité, le moment est-il bien choisi, lorsqu'après deux ans de luttes acharnées l'ennemi est à nos portes, pour susciter des querelles d'allemands à propos de questions futiles [...] ? Parce que certains musiciens français pensent qu'une différence de principe est une raison d'incompatibilité absolue entre eux et leurs confrères, tout projet d' "Union sacrée » doit-il, de ce fait, être écarté [...] ? Que les musiciens qui partagent cette manière de voir se groupent à leur tour, qu'ils s'inspirent de la devise de nos valeureux et héroïques alliés Belges : l'« Union fait la force ", qu'ils forment bloc pour conquérir à la Musique française la place qu'elle mérite d'occuper dans le Monde (Bertelin 1917a, p. 136-137). 
La position de Bertelin est représentative de la ligne éditoriale du Courrier musical en temps de guerre : 1'Union sacrée est une condition cruciale de la réussite de la propagande en faveur de la musique française.

Parmi les aspects les plus évidents de cette propagande figurent les anecdotes et les récits de résistance patriotiques des interprètes et compositeurs français. En juin 1918, par exemple, le correspondant du Courrier musical à Zurich raconte comment, à l'occasion de "représentations de propagande " de Pelléas et Mélisande de Claude Debussy et de Werther de Jules Massenet, « le directeur allemand du théâtre a eu le toupet de tendre la main à M. Salignac ", qui la lui refusa dans un "mouvement spontané et bien français ». Le chanteur proteste également contre la germanisation de l'orthographe des noms des interprètes français sur l'affiche du concert, et contre la présence de nombreux allemands dans l'orchestre et dans les chœurs.

À ce genre de récits, privilégié dans les entreprises de propagande, viennent s'ajouter des slogans. Absents de la revue en temps de paix, ils y font leur apparition

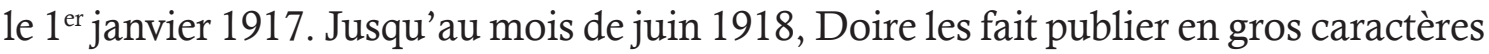
dans les dernières pages des numéros de sa revue, en lieu et place des traditionnels encarts publicitaires (voir figure 1).

\begin{tabular}{|c|c|}
\hline $1^{\text {er }}$ janvier 1917 & Emmenez toujours plusieurs personnes avec vous au concert. \\
\hline 15 janvier 1917 & $\begin{array}{l}\text { Ne vous fâchez pas avec ceux qui prétendent que la musique est déplacée } \\
\text { actuellement, mais convertissez-les. }\end{array}$ \\
\hline $1^{\text {er }}$ février 1917 & $\begin{array}{l}\text { La musique est pour un pays un puissant moyen de propagande, nos } \\
\text { ennemis s'en servaient volontiers. }\end{array}$ \\
\hline 15 février 1917 & $\begin{array}{l}\text { Ne perdez pas votre temps à discuter sur le cas Beethoven ou sur le cas } \\
\text { Wagner: jouez, faites jouer, faites entendre de la Musique française. }\end{array}$ \\
\hline $1^{\mathrm{er}}$ mars 1917 & $\begin{array}{l}\text { Marraines, faites comprendre à vos filleuls, la niaiserie des chansons de } \\
\text { cafés-concerts, indignes des héros. }\end{array}$ \\
\hline $1^{\text {er }}$ avril 1917 & ... vous avez tort! Les pianos français sont supérieurs. \\
\hline Juillet-août 1917 & $\begin{array}{l}\text { Distinguons les Allemands des Boches. Herr Strauss et ses contemporains } \\
\text { seraient aussi mal fondés à se réclamer de Schumann ou de Mozart que ne } \\
\text { l'eût été Tropmann s'il se fût écrié, au bord de l'échafaud: épargnez-moi, car } \\
\text { je descends de Voltaire et de Montesquieu! }\end{array}$ \\
\hline $1^{\text {er }}$ janvier 1918 & $\begin{array}{l}\text { En Allemagne, le Gouvernement alloue à la Maison Krupp } 20 \text { millions pour } \\
\text { organiser la Propagande musicale considérée comme une arme de guerre. } \\
\text { Que compte faire Monsieur Clémenceau après ce que n'ont pas fait ses } \\
\text { prédécesseurs? }\end{array}$ \\
\hline 15 avril 1918 & $\begin{array}{l}\text { Quand la Presse quotidienne française sera-t-elle aussi accueillante pour la } \\
\text { "Grande" Musique, qu'elle l'est pour la "Petite"? Quand la Presse } \\
\text { quotidienne française cessera-t-elle de favoriser presque exclusivement le } \\
\text { "Café-Concert" ennemi de toute pensée élevée, foyer pernicieux de légèreté } \\
\text { et d'absurdité? }\end{array}$ \\
\hline Juin 1918 & $\begin{array}{l}\text { La propagande par la musique - officiellement et intensément pratiquée } \\
\text { chez nos ennemis - est, chez nous, particulièrement délaissée. L'initiative } \\
\text { privée doit intervenir vigoureusement. Que tous les Français désireux de } \\
\text { s'intéresser à cette question d'urgente actualité, veuillent bien nous faire } \\
\text { part de leurs idées, de leurs projets, du genre de collaboration qu'ils seraient } \\
\text { disposés à donner. Nous nous efforcerons de grouper ces nouveaux } \\
\text { éléments de Victoire, au lieu de les laisser s'affaiblir dans l'isolement. Avis: } \\
\text { emportez de la Musique Française pour vos vacances. }\end{array}$ \\
\hline
\end{tabular}

Figure 1 : Slogans publiés dans Le Courrier musical pendant la Première Guerre mondiale. 
Ces slogans permettent de prendre la mesure de la diversité thématique du discours de mobilisation culturelle qui imprègne les articles publiés dans Le Courrier musical pendant la guerre. Elles concernent en particulier l'encouragement de l'économie du concert, de l'édition et de la facture musicale ; la dénonciation de la carence de la politique musicale de l'État, jugée nécessaire à l'entretien des sentiments patriotiques ; la promotion d'une identité musicale française "dégermanisée » et le dénigrement des filiations nationales auxquelles prétendent les compositeurs allemands contemporains. La plupart des articles publiés dans Le Courrier musical de décembre 1916 à novembre 1918 reprennent et développent ces thématiques économiques et esthétiques.

\section{Défendre les intérêts économiques du monde musical français face à l'Allemagne}

Dans le domaine économique, les sujets sur lesquels se mobilise Le Courrier musical concernent au premier chef le secteur privé de l'édition musicale et la facture d'instruments. Dans les deux cas, la concurrence allemande est considérée comme une invasion économique qu'il faut combattre afin d'assurer l'indépendance du pays.

Face à la place importante occupée sur le marché français par des éditeurs allemands tels que Peters, Breitkopf et Härtel ou encore Simrock, Le Courrier musical ne cesse de plaider en faveur de la réalisation par les éditeurs français d'une « édition à bon marché des œuvres classiques » (Poueigh 1916, p. 8). Celles-ci ne sont disponibles que dans des éditions allemandes, alors considérées comme une force d'invasion, au même titre que l'armée :

Bien avant l'heure où éclatait la guerre sanglante, les maisons d'édition allemandes de musique, très décidées d'ailleurs à écarter le plus possible l'art français, avaient établi en France et dans le monde entier les assises prépondérantes de leur commerce [...]. Nos ennemis s'organisèrent à loisir et gagnèrent du terrain. En présence de cet événement, où en est le commerce français ? Qu'a-t-il fait pour regagner le terrain conquis ? Où en est l'édition française invitée par l'une des conséquences brutales du cataclysme mondial à reprendre une place perdue ? Qu'ont réalisé nos éditeurs, véhicules de la pensée artistique, pour remplacer un produit ennemi par une production nationale [...] ? En d'autres termes, la victoire escomptée de nos armes allait-elle doter la France, «terre classique de l'éducation artistique et intellectuelle ", de livres bien à nous, de textes purifiés, portant l'empreinte de notre goût (Tenroc 1917a, p. 54-55).

À travers les articles de Tenroc, la revue relaie des revendications de la Ligue nationale pour la défense de la musique française. Explicitement protectionnistes, celles-ci finissent par avoir des conséquences concrètes : même si le projet d'une édition nationale des classiques n'aboutit finalement pas, des éditeurs français, à l'image de Durand, étoffent leur collection de compositeurs classiques allemands (pour une étude détaillée consacrée à ce sujet, voir Moore 2014).

Une même logique gouverne les revendications pour une réaction des facteurs d'instruments français, à la faveur de la guerre, contre leurs concurrents allemands. Tenroc évoque à ce sujet une "lutte industrielle, véritable guerre " menée par l'Allemagne au moyen d'une " production effrénée, à une propagande inouïe, aux diffusions instituées par des publicités gigantesques »(Tenroc 1917c, p. 385-390). 
Tout en militant pour un redressement de l'industrie de l'édition et de la facture musicale française face à sa concurrente allemande, Le Courrier musical sollicite à de nombreuses reprises les pouvoirs publics et en particulier les deux tutelles de la politique culturelle du pays : le sous-secrétariat aux Beaux-Arts et le ministère de l'Instruction publique.

Les revendications du Courrier musical portent sur la présence de créations d'œuvres françaises modernes dans les programmes de concert. Tout en soutenant les programmes des "Matinées nationales ", manifestations patriotiques inaugurées par Alfred Cortot le 29 novembre 1914, dont les programmes sont dominés par la musique française (Anselmini 2013, p. 150), la revue réclame un conditionnement plus strict du versement des subventions publiques à chaque association de concert au respect du quota d'œuvres françaises inédites (trois heures par saison au moins) imposé par le cahier des charges du sous-secrétariat d'état aux Beaux-Arts :

Malgré les objurgations de notre collaborateur Gaston Carraud, elles [les associations de concert] ne courent aucun risque en exécutant des programmes composés d'ouvrages connus, archi-connus, de tout repos [...]. Pourquoi [le ministre de l'Instruction publique et le rapporteur du budget des Beaux-Arts] ne nommeraientils pas une Commission de musiciens qualifiés, chargée de vérifier l'emploi réel de la subvention? Quel beau rôle l'État aurait ainsi à jouer dans l'effort attendu vers les progrès de la musique, dans la découverte et dans l'essor des jeunes talents de nos compositeurs ! Et combien efficacement il prouverait ainsi qu'il a le souci de ne pas laisser détourner de leur sens les libéralités destinées aux plus nobles buts nationaux! (Puget 1918, p. 28-30).

Le Courrier musical fait également appel à l'État dans le domaine de la diplomatie culturelle. Au moment d'interpeller le sous-secrétaire d'État des Beaux-Arts au sujet de l'organisation des tournées d'interprètes à l'étranger afin de promouvoir l'image de la France, les collaborateurs du Courrier musical citent l'exemple allemand pour mieux signifier l'urgence de passer à l'action, à l'image de Georges Delamare :

Il suffirait, par exemple, d'énumérer les engagements solennels que prirent tour à tour une quantité de ministres, directeurs et sous-secrétaires d'État des BeauxArts sous la troisième République, d'ouvrir la musique française, par d'intelligentes initiatives, des horizons jusqu'ici inconnus [...]. L'Allemagne, elle qui ne néglige aucun facteur de propagande, inonde les pays neutres de tournées symphoniques et nous la regardons faire sans même paraître nous douter qu'il existe une école musicale française (Delamare 1918, p. 279-281).

L'enjeu de ces tournées est simple : la France doit «faire rayonner sur le Monde le pur éclat de sa pensée ». Dans cette lutte d'influence à l'étranger, une compétition est engagée avec l'adversaire allemand. Dans son article, Delamare semble ne pas tenir compte de 1'action du Service de propagande artistique, créé le $1^{\text {er }}$ mai 1916 par le ministère de l'Instruction publique et des Beaux-Arts, à l'initiative d'Alfred Cortot. En 1917, celui-ci organise par exemple six concerts de musique française moderne lors du festival de Barcelone, ou encore une tournée de l'Orchestre de la Société des concerts du Conservatoire en Suisse, du 26 mars au 2 avril (Anselmini 2013, p. 154-155). Cette partialité peut s'expliquer par la ligne éditoriale du Courrier musical: 
rappeler sans relâche que la musique doit être envisagée par les autorités françaises comme une dimension à part entière de la guerre en cours et militer pour une participation toujours plus importante des pouvoirs publics. Toutefois, Delamare ne semble pas surestimer les efforts allemands : l'historien Jean-Claude Montant a montré que, dans le domaine de la presse, les pouvoirs publics allemands ont fait de la propagande une véritable arme auxiliaire, bien avant leurs homologues français et avec plus d'implication (Montant 1988).

Comme dans le domaine des subventions publiques et des tournées à l'étranger, Le Courrier musical milite activement pour que l'État fasse de la musique une véritable cause nationale en encourageant la pratique de la musique à des fins patriotiques dans les écoles publiques (Doire 1917, p. 269). La récurrence et la virulence de ce militantisme sont à la mesure de la faiblesse de l'intervention et du financement étatiques dans le domaine de la propagande musicale (en dehors de l'action menée à l'étranger par le Service de propagande artistique et du soutien accordé en 1916 par le sous-secrétaire d'État aux Beaux-Arts, Albert Dalimier, aux Festivals de musique française [Duchesneau 1996, p. 128]). Afin de donner plus de crédibilité à ses revendications, la revue signale de manière lancinante le retard pris par la France dans le soutien à sa propagande musicale c'est-à-dire - pour rester fidèle à la signification de cette expression dans les années 1910 - à sa politique de diffusion de sa musique et de ses interprètes à l'étranger. En février 1918, par exemple, un article du correspondant du Courrier musical à Berne déplore que :

La propagande musicale allemande se multiplie à Berne sous les formes les plus diverses et il faut ajouter les plus efficaces. Le chef du service de la propagande française est-il au courant ? Sans douter de la bonne volonté de M. René Jean, pouvons-nous espérer que les moyens dont il dispose puissent combattre utilement les efforts de nos ennemis sur le terrain artistique ? Souhaitons-le. Les Bernois, sympathiques à notre art, ne demanderaient qu'à le soutenir. Encore faudrait-il leur en fournir les occasions (Anonyme 1918a, p. 59).

Dans tous les articles qui alertent les lecteurs du Courrier musical sur la situation économique du monde musical français, on retrouve un procédé caractéristique de la rhétorique propagandiste : légitimer la nécessité d'une réaction en insistant sur le danger que représente l'efficacité de l'action de l'ennemi - en l'occurrence, le gouvernement allemand et sa propagande musicale active. Cette rhétorique est également présente dans les slogans cités plus haut (voir figure 1). Elle dénonce le trop faible intérêt manifesté par le gouvernement français à l'égard de la musique. Qu'elle soit favorisée par un secteur privé puissant ou par des politiques publiques, l'influence de la musique allemande en France est ainsi présentée comme une invasion contre laquelle la France doit se défendre. C'est pourquoi la nécessité d'une " dégermanisation » du monde musical est au cœur des revendications du Courrier musical de 1916 à 1918.

Culture et Kultur : une distinction au service de revendications protectionnistes

Dans le domaine des programmes de concerts, la "dégermanisation " se traduit par des revendications en faveur de l'exclusion des œuvres de compositeurs allemands 
tels Anton Bruckner, Gustav Mahler, Max Reger et Arnold Schoenberg, mais aussi et surtout Richard Strauss. Leurs prédécesseurs ne sont pas concernés. Ces choix sont motivés par des arguments économiques avant tout, comme le rappelle le compositeur et critique musical Gaston Carraud :

Portons donc la discussion sur le terrain économique et elle s'éclaircira. Ce que nous ne pouvons admettre, c'est l'idée de procurer chez nous, de longtemps, un gain quelconque à un Allemand vivant [...]. Confisquons à notre profit les maîtres anciens qui n'appartiennent plus qu'au patrimoine commun du progrès humain : jouons-les [...] et laissons un peu à la porte les contemporains (Carraud 1917, p. 216).

Les concerts doivent permettre aux compositeurs français vivants de jouir des droits d'auteurs liés aux performances de leurs œuvres. Jouer la musique de compositeurs allemands contemporains ou récemment décédés impliquerait de verser des droits à l'Allemagne. Telle configuration ne s'applique pas aux "maîtres anciens " qui appartiennent au domaine public, c'est pourquoi, à l'image de Carraud, les tenants de l'argumentaire économique ne visent pas Bach, Mozart et Beethoven, ni même Schubert, Mendelssohn, Brahms et Wagner, alors même que ces derniers sont érigés en symboles de la mère patrie dans la propagande de guerre musicale et illustrée allemande, au même titre que Goethe et Schiller (Giesbrecht 2014, p. 83-102).

Motivée par des raisons économiques, la " dégermanisation » des programmes français recherche toutefois une légitimité en s'appuyant sur des justifications esthétiques et culturelles. Celles-ci s'appuient sur une opposition alors largement répandue dans la presse et dans les milieux intellectuels français et alliés attachés à faire de la Première Guerre mondiale un conflit de civilisation dont toute la responsabilité incomberait à l'Allemagne (voir à ce sujet Prochasson et Rasmussen 1996, p. 203-221 [chapitre "Au service de la nation. L'engagement des savants »] et Temkin 2015). En transposant cette opposition dans le domaine musical, musicographes et critiques musicaux lui confèrent une dimension originale. Sous leur plume émerge une séparation stricte entre une "Kultur » musicale germanique lourde et boursouflée, associée aux compositeurs allemands vivants, et une " culture » musicale classique et universaliste, développée en France et compatible avec l'esthétique des compositeurs germaniques alors considérés comme « classiques ", de Bach à Beethoven en passant par Mozart et Haydn. Régulièrement mobilisée dans les colonnes de la revue, cette opposition trouve sa formulation la plus nette sous la plume de Bertelin dans le premier numéro de guerre du Courrier musical:

Il me parait donc que les œuvres musicales allemandes du XVIII et des deux premiers tiers du XIX ${ }^{\mathrm{e}}$ siècle ont subi à des degrés variables l'influence de notre culture classique et que c'est la raison pour laquelle elles furent si facilement accessibles aux auditeurs français malgré l'essence nettement germanique des thèmes dont elles sont issues. Mais, peu à peu, au cours du XIX ${ }^{e}$ siècle, l'Allemagne, obéissant à la voix des patriotes qui l'appelaient à secouer le joug étranger, s'appliqua à exalter systématiquement tout ce qui était d'essence purement allemande, à dénigrer systématiquement tout ce qui était d'essence latine ou française [...]. Le besoin de dominer, d'opprimer, une mégalomanie jointe à un orgueil insensé ont doté l'Allemagne contemporaine d'un art musical grandiloquent et vide : ces compositeurs hantés par le «Kolossal» 
ne connaissent plus ni proportion ni équilibre [...]. Cet art bien représentatif de leur état d'esprit actuel est la résultante fatale de leur "Kultur». Tout, dans ces monuments monstrueux, [...] est fait pour choquer notre délicatesse, notre sens de la mesure et de l'élégance [...]. Efforçons-nous de sauvegarder nos qualités natives, de développer notre génie propre (Bertelin 1916, p. 5-7).

La distinction de Bertelin est reprise dans Le Courriermusical par Tenroc (Tenroc 1918a), d'Indy (D'Indy 1917) ou encore la musicologue Marie Bobillier (sous le pseudonyme de Michel Brenet, Brenet 1917). Elle repose tout d'abord sur une ethnicisation des styles nationaux : il existe par exemple une " essence germanique " des thèmes musicaux. L'évolution des styles est toutefois perméable aux évolutions historiques : c'est à ce titre que Bertelin fait de l'évolution de la musique allemande une métaphore de la montée du pangermanisme belliqueux et impérialiste, alors considéré en France comme la cause principale de la guerre. L'Allemagne contemporaine est dangereuse, car elle s'est débarrassée de toutes les influences de la culture « d'essence latine ou européenne " au profit d'une Kultur purement germanique, gonflée d'un " orgueil insensé », " mégalomane » et " monstrueuse », pour reprendre les termes de Bertelin. Ces qualificatifs, que l'on retrouve dans la propagande antiallemande (voir figures $2 \mathrm{a}$ et $2 \mathrm{~b}$ ), contribuent à justifier les appels au boycott des compositeurs modernes contemporains allemands dans les programmes de concerts français, Wagner et Strauss en particulier.

Figure 2a: Henry Cold, Le pou. Graine de boches $-\mathrm{G}^{\mathrm{de}}$ Kultur, «Semence : 1914 »(carte postale), Nice, 1914.

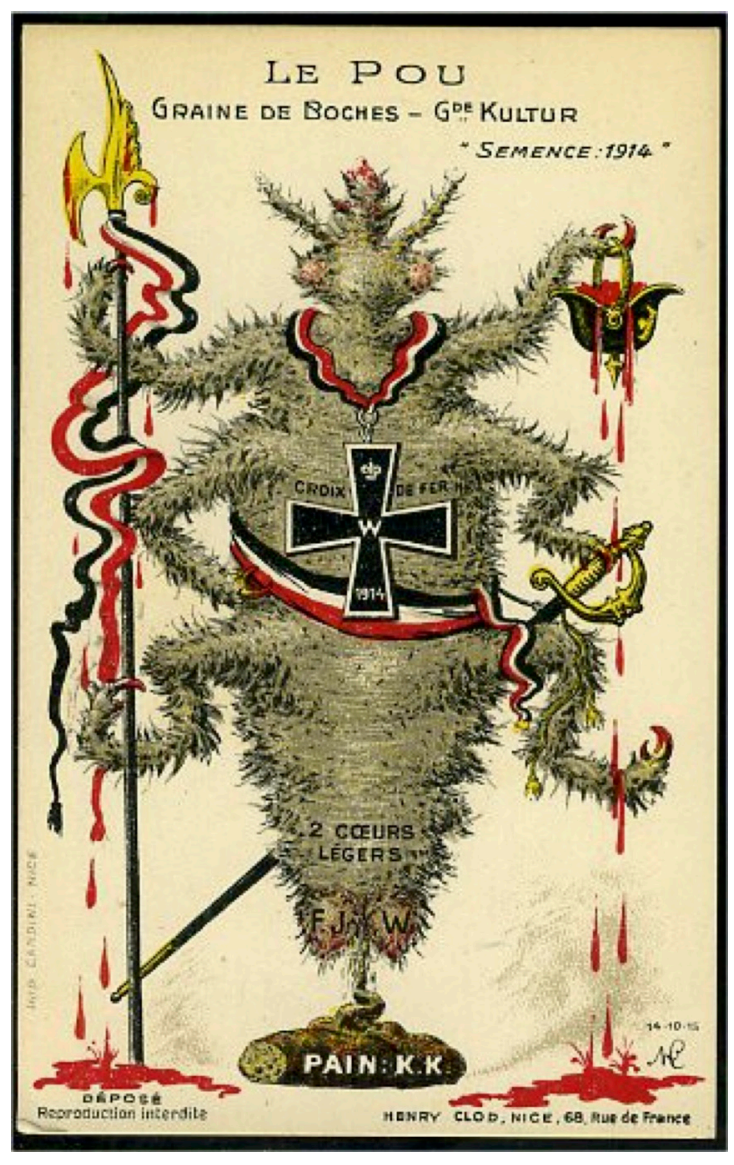

Revue musicale OICRM, volume $4, n^{\circ} 2$ 


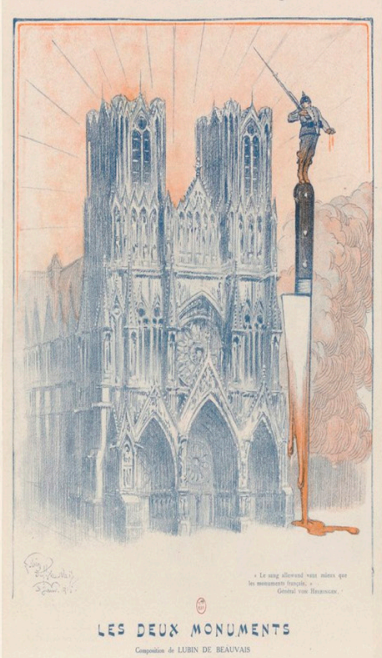

Figure $2 b$ : Lubin de Beauvais,

"Culture et Kultur», Fantasio, septembre 1915.

La notion de Kultur permet en effet d'assimiler la musique allemande contemporaine à une menace pour l'intégrité de la musique française. Celle-ci se voit en retour renvoyée à un "génie propre ", fait de valeurs classiques : "proportion ", " équilibre ", " mesure " et " élégance ", pour reprendre les termes de Bertelin. Plus largement, la guerre implique pour certains musiciens ressortissants des pays alliés « la volonté d'affirmer contre l'invasion allemande et du Nord l'immortelle vitalité de l'âme latine » (Gatti 1917, p. 69).

Les articles qui viennent d'être cités permettent de dégager les principaux axes de la ligne éditoriale défendue par Le Courrier musical au nom d'une Union sacrée $\mathrm{du}$ monde musical, au nom de ses propres intérêts économiques et du développement d'une musique libérée des influences germaniques contemporaines. Il convient toutefois de revenir sur cette Union. De même qu'elle ne perdure pas pendant toute la guerre dans le monde politique et dans la société françaises, des débats et des dissensions se font jour dès 1917 au sein même du Courrier musical.

L'Union sacrée jusqu'au bout? La place des voix dissidentes

Premièrement, Doire continue de faire respecter dans la revue le droit de réponse et de démenti. Un mois après la parution du compte rendu du geste patriote et héroïque de M. Salignac à Zurich, un démenti catégorique de Salignac lui-même est publié dans Le Courrier musical (Anonyme 1918c, p. 270). 
Deuxièmement, le nationalisme jusqu'au-boutiste et les positions les plus radicales prises contre la musique allemande font l'objet de débats contradictoires au sein de la revue. Lorsque Saint-Saëns, emporté par le violent anti-germanisme qu'il exprime notamment dans L'Écho de Paris, accuse d'Indy de traîtrise au prétexte que ce dernier n'est pas hostile au retour des œuvres de Wagner sur les scènes françaises (pour une étude détaillée de la place de Wagner dans les programmes de concerts français pendant la guerre, voir Buch 2004), Doire répond en août 1917 que :

Cette fois encore, L'Écho de Paris a généreusement ouvert ses colonnes à l'auteur de Samson pour lui permettre de développer ses rancœurs et ses rancunes. M. SaintSaëns s'est fâcheusement entraîné depuis la guerre à écrire dans un sens violent et irréfléchi [...]. Ajoutons pour nous " réhabiliter » aux yeux de ceux qui s'apprêtent à nous taxer de mauvais Français, que nous sommes parfaitement d'accord avec M. Saint-Saëns sur le silence dans lequel nous devons persister autour des œuvres allemandes contemporaines. Mais encore une fois Wagner n'est pour rien dans le conflit actuel, et ses œuvres, sur l'esprit national desquelles nous reviendrons volontiers, sont tombées dans le domaine public. C'est donc se donner bien du souci que de brûler ses partitions, ainsi que le si éminent Frédéric Masson nous y a conviés à plusieurs reprises! (Doire 1917, p. 267-269).

Le nationalisme du Courrier Musical est plus modéré que celui de Saint-Saëns et de La Musique pendant la guerre. Il se limite en effet à saisir l'opportunité offerte par la période d'exception que constitue la guerre pour renforcer la situation économique $\mathrm{du}$ monde musical français et concurrencer plus efficacement celui de l'Allemagne. Selon l'argumentaire de Doire, proche de celui de Carraud évoqué plus haut (Carraud 1917, p. 216), la musique de Wagner ne représente pas un danger dans la mesure où, depuis le mois de juillet 1914, elle est effectivement tombée dans le domaine public. Doire invalide ainsi la partie économique de l'argumentaire des opposants au retour du compositeur de Siegfried dans les programmes français.

Pendant la guerre, Le Courrier musical demeure même ouvert à une critique du nationalisme musical. Le 15 juillet 1918, le compositeur Alfredo Casella peut ainsi affirmer en première page que "ce problème [le nationalisme musical] est absolument accessoire auprès de celui - infiniment plus important - qui concerne la beauté, l'originalité, la valeur finale de l'œuvre d'art. La première, la seule raison d'être de toute œuvre d'art, est d'être neuve [...] et belle »(Casella 1918, p. 77-79). La mobilisation du Courrier musical et les appels à l'Union sacrée n'entraînent donc pas la censure des positions qui s'écartent de la ligne défendue par Doire, Tenroc et Bertelin, une ligne située entre l'extrémisme de Saint-Saëns et le refus du nationalisme défendu par Casella. L'exemple du Courrier musical tend donc à confirmer la tendance à la « réintroduction du débat » identifiée à partir de l'année 1916 par Charlotte SegondGenovesi, dans son étude sur la Gazette des classes du Conservatoire (SegondGenovesi 2009, p. 184). Ce même exemple incite toutefois à nuancer l'interprétation qu'en propose la musicologue, selon laquelle la polarisation des prises de position tendrait à recouper une « la dichotomie civil-soldats », la « séparation entre [musiciens de] l'arrière et [du] front " (Segond-Genovesi 2009, p. 187). Bien réelle dans le cas des élèves et anciens élèves du Conservatoire de Paris, la correspondance entre divergences musicales et l'opposition front/arrière est beaucoup moins nette chez les 
collaborateurs du Courrier musical, signe que la rupture de l'Union sacrée se traduit également par des divisions au sein de la population demeurée à l'arrière. En témoignent les divergences entre Charles Koechlin, resté malgré lui à l'arrière, cherchant à composer "sans haine et sans ignorance de l'étranger » (Koechlin 1918, p. 107), réclamant contre "l'épithète : "style boche" " (Koechlin 1917, p. 79) et dénonçant indirectement les prises positions nationalistes de la Ligue nationale pour la défense de la musique française (voir à ce sujet Caillet 2009, p. 126-128). En témoigne également l'infrangible union de Ravel, qui a fait l'expérience du front, et d'Émile Vuillermoz, lui aussi mobilisé mais à Paris, loin des combats. En 1916, tous deux s'opposent de concert aux tentatives de réunification menées par Cortot au nom de 1'Union sacrée (Duchesneau 1996, p. 133-137). Ravel et Vuillermoz considèrent que "l'art ne s'accommode pas des mêmes principes que la politique " (Vuillermoz 1916). C'est pourquoi la démarche de Cortot consistant à appliquer les principes de l'Union sacrée à la musique relève pour eux de l'" assassinat ».

Jamais plus qu'en 1916, 1917 et 1918, Le Courrier musical n'a privilégié les articles consacrés aux oppositions économiques, culturelles et esthétiques entre France et Allemagne. Ces trois années de guerre constituent à ce titre une période singulière dans l'histoire de la revue. Toutefois, elles ne marquent ni une rupture radicale avec les années précédant la guerre, ni une parenthèse qui se refermerait une fois signé l'armistice.

Au-DelÀ du CONFLit : ENTRÉE ET SORTIE DE GUERRE DU COURRIER MUSiCAL

Une mobilisation culturelle amorcée avant la guerre : nationalisme et anti-germanisme

Le discours développé pendant la guerre par Le Courrier musical ne fait que réactiver et développer avec une intensité inédite une rhétorique et des thématiques déjà à l'œuvre pendant la décennie 1900. L'un des exemples les plus frappants de cette continuité réside dans l'essentialisation de l'opposition entre une sensibilité musicale française considérée comme équilibrée, classique et une sensibilité allemande présentée comme brutale et grandiloquente. Cette opposition émerge dans la revue dix ans avant le début de la guerre. Nourrie par l'esprit revanchard de 1870, elle est concomitante au réveil des tensions entre la France et l'Allemagne à propos de la question marocaine. À partir de 1904 en effet, une série d'articles de Bertelin, Gaston Knosp, Lionel de La Laurencie, Camille Mauclair et Paul de Stœcklin met en circulation dans Le Courrier musical l'idée selon laquelle la musique allemande moderne reflèterait les velléités militaristes et impérialistes allemandes (Bertelin 1912 et 1913, La Laurencie 1904, Knosp 1905, De Stœcklin 1911 et Mauclair 1911). Germaniste et spécialiste des cultures nordiques, De Stœcklin les décrit explicitement :

Le moteur essentiel de tout le mécanisme germanique est [...] le chauvinisme organisé qui en active tous les rouages. À l'état de force potentielle dans les siècles passés, cet esprit s'est manifesté sous le premier empire par la haine affichée et, depuis 1870, par un envieux dédain de tout ce qui n'est pas allemand [...]. Notre tradition française est logique et normale. Elle représente ce que le passé nous transmet de vivant et de conforme à notre génie [...]. Nos voisins d'Outre-Rhin ont [...] surtout la religion 
du grand homme pour la gloire duquel la nation ne saurait faire trop de sacrifices. Car le grand homme répand l'éclat de sa race et affirme sa supériorité, il est la clé de voûte au moyen de laquelle se construira l'édifice du germanisme universel. L'âme allemande devient envahissante, et son trop-plein d'activité se répand dans tous les domaines (De Stœcklin 1904a, p. 502-503).

Entre les mains des Allemands, la [musique] fut [...] le moyen d'expression du vague sentimental [...], de la sensibilité métaphysique, dont Wagner fit une arme de combat et de pénétration (De Stœecklin 1904b, p. 525).

De l'impérialisme à l'arrogance en passant par le conservatisme et le militarisme, le stéréotype racial du "Boche ", élément central de la propagande française et alliée (Buitenhuis 1987, p. xvi) est donc en place dans Le Courrier musical bien avant 1914 (la genèse de ce stéréotype à partir de la guerre de 1870 a été étudiée dans Jeismann 1992). Il joue de plus la même fonction en 1904 qu'entre 1916 et 1918 : persuader le monde musical français de la nécessité d'une réaction urgente face à une situation décrite comme une invasion de la musique allemande en France. Là encore, les considérations historiques et esthétiques cachent une rivalité avant tout économique. L'objectif des collaborateurs cités plus haut consiste à défendre les "parts de marché » d'une musique française qu'ils estiment dangereusement concurrencée par la musique allemande. Le Courrier musical contribue ici à transposer dans le domaine de la musique les craintes suscitées en France par le développement de la puissance commerciale de l'Allemagne et incarnées par la figure mythifiée du commis-voyageur (Marin 2012). Certains appels à une réaction contre la musique allemande contemporaine témoignent même d'une entrée en guerre avant l'heure de certains critique musicaux : en 1911, Mauclair dénonce l'engouement du public français pour Mahler et Strauss au moyen d'une métaphore filée de la guerre francoallemande. Cet engouement est présenté comme le résultat d'un « assaut » des deux compositeurs :

Il y a du houzard [hussard] chez Mahler et du cuirassier blanc chez Strauss [...]. Il est significatif que l'Europe centrale s'émeuve à la voix rude de symphonistes qui écartent le concept de beauté, dans le pays de Mozart-Raphaël et de BeethovenMichel-Ange, pour tâcher de construire, avec des sonorités, des sortes de balistes, d'hélépoles, de béliers monstrueux, afin d'enfoncer les portes des consciences et de broyer les âmes collectives des foules sous une musique frénétique glorifiant le dieu farouche de la Brutalité de la Guerre (Mauclair 1911, p. 120-121).

La distinction entre compositeurs allemands qui relèvent de la Kultur et artistes germaniques compatibles avec l'esprit français est également en place avant la guerre. De Stœcklin considère par exemple que Schiller et Goethe, au même titre que Beethoven, sont "fécondés tous deux par 1'art antique, par 1'art classique » (De Stœcklin 1911, p. 44). Leurs productions ne peuvent donc être rattachées à ce " germanisme » belliqueux qui, selon De Stœcklin s'est développé à partir de 1870 (De Stœcklin 1904a).

Dès les années 1900, Le Courrier musical contribue donc à mobiliser du monde musical français contre l'influence de son voisin germain, perçu comme une menace. Plutôt que de susciter de nouveaux discours, la Première Guerre mondiale joue un 
rôle de catalyseur : elle permet de faire émerger au premier plan des débats et des prises de positions déjà lancés en amont. Le conflit militaire entre la France et 1'Allemagne crée un effet d'aubaine pour les défenseurs de ces positions, en donnant plus de poids à ses revendications et en permettant à certaines d'entre elles d'aboutir. De fait, les années 1915-1918 sont celles d'un recul sensible de la place des compositeurs allemands vivants dans les programmes des concerts en France (voir à ce sujet Leterrier 2000 et Buch 2004). C'est aussi pendant la guerre que l'État choisit d'intervenir de manière directe dans la diffusion de la musique française à l'étranger.

De même que le discours de mobilisation culturelle du Courrier musical précède de près de dix ans le déclenchement du conflit, elle ne cesse pas avec la fin des hostilités. Les contenus publiés par la revue dans les années qui suivent la Première Guerre mondiale sont un excellent indicateur de la lente démobilisation culturelle d'une partie du monde musical français.

Une guerre "qui ne passe pas » : la lente démobilisation culturelle du Courrier musical

«Le cliquetis des armes s'est éteint [...]. La vie a repris ses droits » (Nin 1919, p. 293-294). "La Musique [...] retrouve la sérénité de sa liturgie bousculée » (Tenroc 1919a, p. 97). À lire le compositeur cubain Joaquin Nin et Tenroc, une page semble s'est tournée en 1919. La guerre est présentée comme une parenthèse dont la fermeture permet un retour à la normalité. Ce retour à la normale se manifeste par une augmentation sensible du nombre d'articles consacrés à la musique, indépendamment de toute considération pour la situation politique et géopolitique (voir, entre autres, Huré 1919a et 1919b, Stany 1919 et Simon 1921). De plus, la musique allemande naguère associée à la Kultur retrouve une place dans la revue. Léon Vallas justifie ce retour en désolidarisant la valeur esthétique des œuvres musicales du contexte géopolitique : " le nationalisme le plus intransigeant ne peut faire table rase des trésors de peuples justement honnis » (Vallas 1919, p. 83). Au mois de janvier 1919, le dernier slogan publié dans la revue annonce toutefois que si "Le Courrier musical acclame la Paix entre les partis musicaux, [il] déclare la Guerre à ceux qui servent mal la Musique et les Musiciens de France " (Anonyme 1919a). Quoique métaphorique, la permanence de la référence à la " guerre " est le signe d'une démobilisation incomplète au lendemain de la guerre.

Pendant l'année 1919, Le Courrier musical continue en effet d'évoquer le conflit franco-allemand à travers des caricatures. L'une d'entre elles, œuvre de Georges Villa, présente le chef d'orchestre Camille Chevillard " préparant une attaque... » (Villa 1919a ; voir figure 3a). La fumée qui sort des pavillons de tuba et de trombone représentés en arrière-plan les apparente à des canons. Le mois suivant, pour célébrer les 150 ans de la mort de Berlioz, le même Villa le représente «triomphant, sur les bords du Rhin »(Villa 1919b, voir figure 3b). 


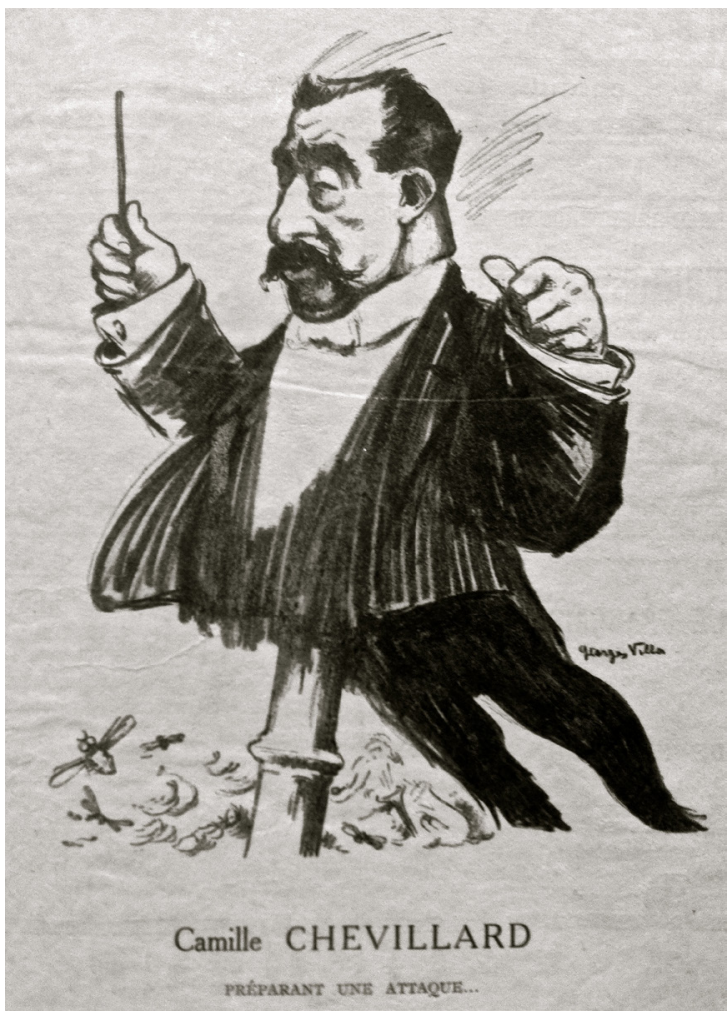

PRḰPARANT UNB ATTAQUE.
Figure 3a: Georges Villa, "Camille Chevillard préparant une attaque...", Le Courrier musical, vol. 21, $n^{\circ} 5$ (1' mars 1919), n. $p$.

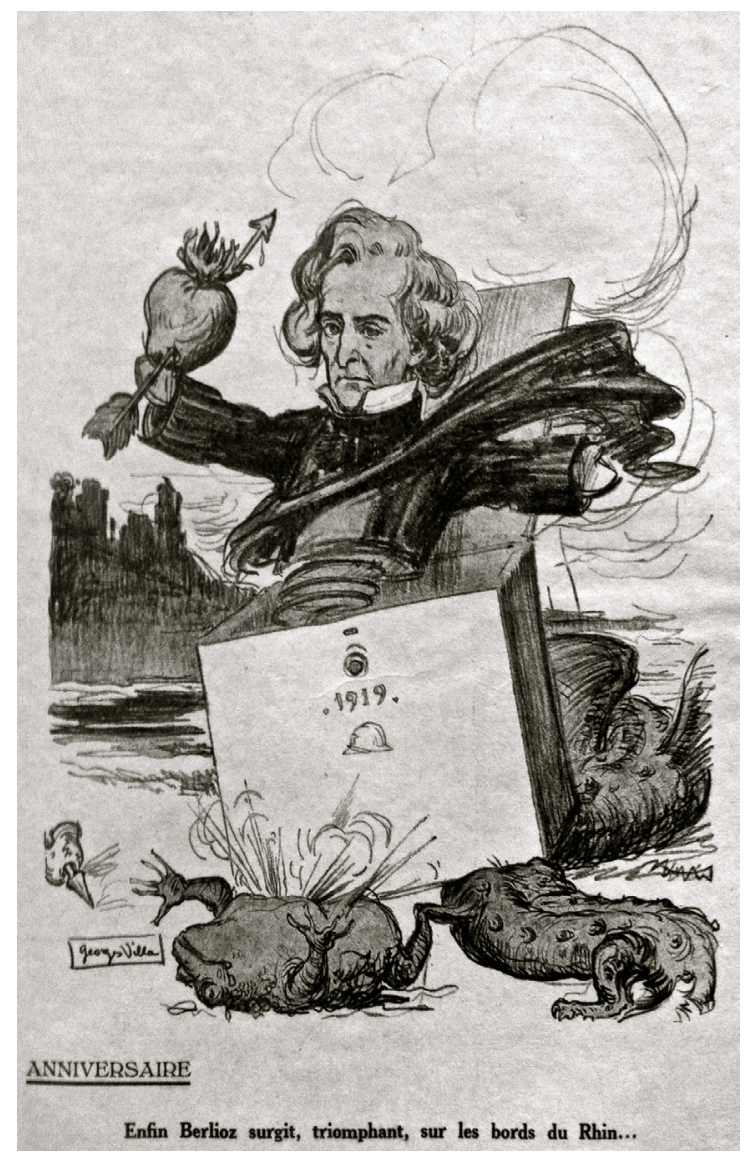

Figure 3b: Georges Villa, "Anniversaire. Enfin Berlioz surgit, triomphant, sur les bords du Rhin...", Le Courrier musical, vol. 21, $n^{\circ} 6$ (15 mars 1919), n. $p$. 
Dans cette caricature, le compositeur français surgit hors d'une boîte surprise et écrase un crapaud et un dragon, deux animaux associés aux Allemands grâce à la présence d'un casque à pointe. Signe qu'en 1919, l'animalisation de l'ennemi, centrale dans la propagande de guerre (Billi 2015, p. 157-170), n'appartient pas encore à un passé révolu. De même qu'une partie de l'opinion française cherche à préserver l'esprit de l'Union sacrée en votant pour le Bloc national de novembre 1919 à 1924, Le Courrier musical reste imprégné pendant toute la première moitié des années 1920 au moins, par un discours situé dans la droite ligne de celui développé pendant la guerre. Il contribue ainsi à la " perpétuation de la mémoire du conflit " étudiée par Jane Fulcher et par Barbara Kelly pour les années 1920 (Fulcher 2005, p. 86-118, Kelly 2013, p. 37-66).

Trois thématiques perdurent sous une forme identique à celle observée pendant la guerre. Premièrement, dans le domaine économique, Le Courrier musical continue de plaider pour une union des intérêts privés des éditeurs français. Pour Louis Vuillemin, l'absence d'union ne peut profiter qu'à l'Allemagne, qu'il considère encore comme un adversaire :

Les Sonates de Beethoven, Mozart, les Symphonies classiques arrangées... [...]. Ces musiques sont introuvables chez nous. Elles ne se trouvent qu'à la condition de faire commerce clandestin avec l'ennemi. Quelle responsabilité, ô éditeurs, mes amis ! Voilà où nous a réduit votre résistance au Consortium de vos forces, à l'union en vue de l'édition nationale (Vuillemin 1919, p. 61).

Tenroc, décoré de la Légion d'honneur avec Mauclair en 1919 pour sa participation à la défense de la musique française pendant la guerre (Anonyme 1919b, p. 222), adopte la même position : l'enjeu de l'" énorme trust, [1']immense office musical » qu'il appelle de ses vœux doit répondre à un objectif unique : le "rayonnement mondial » de la musique française (Tenroc 1919b, p. 115-116).

Deuxièmement, le retour de la paix n'éteint pas les revendications en faveur d'une intervention accrue des pouvoirs publics. "Comment ne pas voir que les intérêts de l'art sont aussi précieux, plus précieux même à la France que ceux du commerce, de l'industrie et de la finance ? ", explique le musicologue et critique Louis Laloy, avant de déplorer que « la subvention [à l'Opéra] n'a jamais été aussi faible que sous la troisième République » (Laloy 1921, p. 89), contrairement aux autres pays européens et aux États-Unis. Laloy emprunte à la rhétorique de la propagande de guerre lorsqu'il cite l'action des gouvernements étrangers en exemple pour donner plus de poids à sa dénonciation de l'incurie des pouvoirs publics français.

Troisièmement, une partie des collaborateurs du Courrier musical continue à faire preuve d'une vigilance extrême quant à la répartition des œuvres françaises et étrangères - allemandes en particulier - dans les programmes de concerts parisiens. L'idée défendue est que la fin de la guerre ne doit pas déboucher sur un statu quo par rapport aux années 1900. L'une des manifestations les plus visibles de cette position est «l'affaire des poisons », déclenchée en 1923 par le critique musical Louis Vuillemin contre le compositeur et organisateur de concerts Jean Wiéner. Désireux de faire connaître au public parisien les œuvres de l'avant-garde musicale, celui-ci programmait régulièrement du jazz américain, mais aussi des œuvres de Schönberg, de Berg et de Webern : 
Oui. Il y a quelque part dans Paris, des "Concerts métèques »! [...] Des fumistes inopportuns, [...] malfaisants [...], s'empressent [...] de découvrir tout ce que le mauvais goût international a produit et l'importent au cœur de la capitale, dans l'évident espoir de le faire battre de travers [...]. L'effort de ces jobards cosmopolites [...] pourvus de lunettes à la boche a pour but, sans doute, de gangréner notre organisme : de démontrer aussi aux étrangers curieux, présents en nombre dans la salle, "l'affaissement du goût chez les Français d'après-guerre »! (Vuillemin 1923, p. 4).

Pour Vuillemin, l'enjeu de la présence d'œuvres contemporaines étrangères rejoint celui de la guerre qui vient de s'achever. À travers une métaphore médicale, elle est assimilée à un danger pour la France, à la menace d'une défaite, à une maladie propagée par des sympathisants de l'ennemi (désigné à travers l'expression « lunettes à la boche »). Cinq ans après la fin des combats, la présence des œuvres étrangères demeure associée à une invasion contre laquelle le monde musical français doit se prémunir. Vuillemin s'inscrit dans la droite ligne des objectifs que Tenroc assignait à la revue un mois après la signature de l'Armistice : poursuivre la « guerre [...] contre les "bocheries" malsaines" (Tenroc 1918b, p. 362). Tenroc et Vuillemin ne sont pas les seuls collaborateurs du Courrier musical à mener ce combat. En 1919, Foulques Nerra continue lui aussi de militer contre "l'invasion forcée des programmes par [...] la musique "Boche", imposée par les "Boches" - Richard Strauss, Brahms, Bruckner, et par-dessus tout l'obsession wagnérienne dont la guerre a débarrassé Paris et le public français » (Nerra 1919, p. 140-141. Foulques Nerra est le pseudonyme d'un critique musical qui n'a pu être identifié).

La permanence du discours de guerre dans Le Courrier musical concerne également la nécessité de dégermaniser certains compositeurs allemands. Déjà opérée à propos de Bach, de Mozart et de Beethoven pendant la guerre, cette dégermanisation s'applique à Wagner à partir de 1919. C'est Tenroc lui-même, l'un des artisans les plus actifs du discours de guerre du Courrier musical, qui prend la plume en 1921 pour saluer le retour de Wagner sur les scènes françaises. La guerre étant terminée, il salue le public qui « a compris la suprême idiotie qui consiste à se replier pour soi-même en se privant d'une belle jouissance esthétique, sous prétexte d'une hypocrisie. Il a compris que la proscription a assez duré " (Tenroc 1921, p. 24). Reste toutefois à justifier un retour qui peut sembler contradictoire avec le discours formulé dans la revue contre la musique allemande moderne. Pour ce faire, les admirateurs assumés de Wagner tentent, dès 1919, de délivrer sa musique du « stigmate spécial d'impérialisme pangermanique " (Mauclair 1919, p. 3) qui lui a été infligé pendant la guerre. Plus précisément, l'habileté rhétorique des commentateurs consiste à retourner ce stigmate contre l'Allemagne, comme le propose le critique Henri Chéronnet :

L'Anneau du Nibelung est-il une épopée nationale allemande ? En rien. Il ne dérive pas des antiques annales de la race, car il tire son sujet beaucoup moins du Nibelungen Lied que des Eddas scandinaves. [...]. La Tétralogie est un monument de l'esprit humain, elle peut prendre place à côté de L'Orestie, de La Divine comédie, et du théâtre de Shakespeare. C'est en vain que la Germanie moderne, cruelle et sadique dans le triomphe, lâche et abjecte dans l'adversité, voudrait s'en réclamer [...]. Si les Boches avaient compris ainsi la Tétralogie, comment n'auraient-ils pas vu qu'elle constitue un réquisitoire précis et inexorable de leur conduite au cours des hostilités? (Chéronnet 1919, p. 210-211). 
Érigée en classique, la Tétralogies'adresse à l' «esprit humain ». Sa dimension universelle lui permet d'échapper à toute assignation à une identité nationale particulière. Quand bien même cette assignation devrait être opérée, elle concernerait moins l'Allemagne que la Scandinavie. Mieux : la Tétralogie est mise au service du discours de guerre que perpétue Le Courriel musical jusqu'au début des années 1920, puisqu'elle peut être interprétée comme une condamnation de l'impérialisme germanique.

\section{CONCLUSION}

Le Courrier musical a contribué de manière décisive à mettre la musique et le monde musical savants au service de la mobilisation culturelle de la société française pendant la Première Guerre mondiale. Au-delà des prises de position en faveur d'une lutte contre la concurrence économique des éditeurs de musique et des facteurs allemands, le discours proposé par les collaborateurs de la revue a contribué à essentialiser une opposition entre identité musicale française et allemande, et à ériger cette opposition en métaphore du conflit franco-allemand. À sa manière, Le Courrier musical a donc participé à la propagande antiallemande développée en France pendant la Première Guerre mondiale.

La guerre n'a toutefois constitué qu'une rupture partielle dans l'histoire de la revue. Elle a fourni à ses collaborateurs une occasion pour donner plus de poids à des revendications nationalistes qui résultent d'un processus de mobilisation culturelle engagé dès le début des années 1900. D'autre part, la démobilisation culturelle du Courrier musical ne coïncide pas avec la fin officielle de la Première Guerre mondiale. Sa ligne éditoriale nationaliste renvoie à la permanence de la conflictualité et du sentiment antigermanique dans la France du début des années 1920. La question des réparations et l'attribution de la responsabilité du conflit à la seule Allemagne dans le traité de Versailles ne font qu'alimenter cette conflictualité. En ce sens, musicographes et critiques musicaux apportent une contribution originale aux débats menés hors du monde musical au sujet de la nature de l'opposition culturelle entre France et Allemagne.

Certes, Le Courrier musical, seule grande revue à paraître de 1916 à 1918 ne peut être considéré comme l'unique baromètre du nationalisme qui anime le monde musical français. C'est précisément en réaction avec sa ligne éditoriale qu'est créée en 1920 La Revue musicale d'Henry Prunières, héritière de La Revue musicale sIM, volontiers internationaliste et en tout cas beaucoup plus modérée vis-à-vis de la musique allemande. Quoiqu'il en soit, la revue de Doire et Tenroc nous fournit un cas exemplaire de processus de mobilisation et de démobilisation culturelle de la presse musicale avant, pendant et après un conflit. Elle permet également de confirmer que l'emploi de la musique à des fins de propagande ne se limite pas aux périodes de guerres (voir à ce sujet Sala 2014). Un tel constat ne peut qu'inviter l'historien de la musique à ne pas calquer le découpage chronologique de l'histoire de la musique sur les bornes chronologiques traditionnelles des périodes de paix et de guerre. 


\section{BIBLIOGRAPHIE}

Anonyme (1918a), «Échos. Propagande », Le Courrier musical, vol. 20, [s. no.] (1 ${ }^{\text {er }}$ février), p. 59.

Anonyme (1918b), "Échos. Comment est faite notre propagande à l'étranger ", Le Courrier musical, vol. 20, [s. no.] (juin), p. 237-238.

Anonyme (1918c), «Échos », Le Courrier musical, vol. 20, [s. no.] (juillet-août), p. 270.

Anonyme (1919a), «Notre affiche actuelle », Le Courrier musical, vol. 21, n 2 (15 janvier), [n. p.].

Anonyme (1919b), «Échos », Le Courrier musical, vol. 21, nº 14 (août-septembre), p. 222.

Anselmini, François (2013), "Alfred Cortot et la mobilisation des musiciens français pendant la Première Guerre mondiale ", Vingtième siècle. Revue d'histoire, no 118 (avril- juin), p. 147-157, https://www.cairn.info/revue-vingtieme-siecle-revue-d-histoire-2013-2-page-147.htm, consulté le 3 juillet 2017.

Audoin-Rouzeau, Stéphane, et al. (dir.) (2009), La Grande Guerre des musiciens, Lyon, Symétrie.

Audoin-Rouzeau, Stéphane, et Jean-Jacques Becker (1995), La France, la nation, la guerre (1850-1920), Paris, Sedes.

Baty-Delalande, Hélène, et Carine Trévisan (dir.) (2016), Entrer en guerre, Paris, Hermann.

Becker, Annette (2000), "Musique et culture de guerre », dans Annette Becker et al., Chefs-d'ouvre et circonstances, Arras, Archives départementales du Pas-de-Calais, p. 9-14.

Becker, Jean-Jacques (1977), 1914. Comment les Français sont entrés dans la guerre, Paris, Presses de Science Po.

Becker, Annette, et Jean-Jacques Becker (1988), La France en guerre 1914-1918. La grande mutation, avec la collaboration d'Annette Becker, Bruxelles, Complexe.

Bertelin, Albert (1912), "L'évolution de la musique contemporaine (suite) », Le Courrier musical, vol. $15, \mathrm{n}^{\circ} 19$ (1 $1^{\mathrm{er}}$ octobre), p. 502-506.

Bertelin, Albert (1913), "Le nationalisme en art ", Le Courrier musical, vol. 16, $\mathrm{n}^{\circ} 3$ (1 $1^{\mathrm{er}}$ juillet), p. 362-368.

Bertelin, Albert (1916), "Culture française et Kultur germanique », Le Courrier musical, vol. 18, [s. no.] (1 ${ }^{\text {er }}$ décembre), p. 5-7.

Bertelin, Albert (1917a), «Variations sur un vieux thème», Le Courrier musical, vol. 19, [s. no.] (15 mars), p. 136-137.

Billi, Francesco (2015), Il Racconto Postale della Grande Guerra. Origini della communicazione di massa in Italia e in Romangna. Propaganda e satira nel primo conflitto mondiale, Cesena, Società Editrice «Il Ponte Vecchio ».

Brenet, Michel (1917), «Pour les gloires de l'Italie », Le Courrier musical, vol. 19, [s. no.] (1 $1^{\text {er }}$ janvier), p. 60-62.

Buch, Esteban (2004), " "Les Allemands et les Boches". La musique allemande à Paris pendant la Première Guerre mondiale », Le Mouvement social, $\mathrm{n}^{\circ}$ 208, p. 45-69, https://www.cairn.info/revuele-mouvement-social-2004-3-page-45.htm, consulté le 30 août 2017.

Buitenhuis, Peter (1987), The Great War of Words. British, American and Canadian Propanda and Fiction (1914-1933), Vancouver, University of British Columbia Press.

Caballero, Carlo (1999), " Patriotisme or Nationalisme? Fauré and the Great War », Journal of the American Musicological Society, vol. 52, no 3, p. 595-626.

Cabanes, Bruno, et Guillaume Piketty (2007), "Sortir de la guerre. Jalons pour une histoire en chantier ",Histoire@Politique, vol. 3, no 3, p. 1-12, http://www.cairn.info/revue-histoire-politique2007-3-p-1.htm, consulté le 3 septembre 2015.

Caillet, Aude (2009), "1914-1918. Rupture et renouveau : l'effort de guerre de Charles Koechlin », dans Myriam Chimènes et al. (dir.), La Grande Guerre des musiciens, Lyon, Symétrie, p. 119-133. 
Carraud, Gaston (1917), « Pour la musique française. La musique étrangère en France ", Le Courrier musical, vol. 19, [s. no.] (mai), p. 216.

Casella, Alfredo (1918), "La nouvelle musicalité italienne ", Le Courrier musical, vol. 20, [s. no.] (15 février), p. 77-79.

Chéronnet, Henri (1919), "Wagner contre l'Allemagne ", Le Courrier musical, vol. 21, nº 14 (aoûtseptembre), p. 210-211.

D'Almeida, Fabrice (2002), " Propagande, histoire d'un mot disgracié ", Mots. Les langages du politique, n 69 , p. 137-148, https://mots.revues.org/10673, consulté le 24 mai 2017.

Daudet, Léon (1918), La Guerre totale, Paris, Nouvelle Librairie Nationale, http://gallica.bnf.fr/ ark:/12148/bpt6k67250.r=, consulté le 5 décembre 2016.

De La Laurencie, Lionel (1904), «Au pays de la critique musicale », Le Courrier musical, vol. 7, n 3 ( ${ }^{\text {er }}$ février), p. 78-82.

Delamare, Georges (1918), «Les paroles s'envolent », Le Courrier musical, vol. 20, [s. no.] (septembreoctobre), p. 279-281.

De Stœcklin, Paul (1904a), «La vie musicale en Allemagne. 1. L'âme allemande », Le Courrier musical, vol. 7, $\mathrm{n}^{\circ} 18$ (15 septembre), p. 502-503.

De Stœcklin, Paul (1904b), "La vie musicale en Allemagne. 2. Musique et musique allemande ", Le Courrier musical, vol. 7, $\mathrm{n}^{\circ} 19$ (1 $1^{\mathrm{er}}$ octobre), p. 525.

De Stœcklin, Paul (1911), «Étude sur la musique romantique allemande », Le Courrier musical, vol. 14, $\mathrm{n}^{\circ} 2$ (15 janvier), p. $42-46$.

D’Indy, Vincent (1917), «Encore quelques notes sur la musique », Le Courrier musical, vol. 19, [s. no.] (1 ${ }^{\text {er }}$ novembre), p. 339-342.

Doé de Maindreville, Florence, et Stéphan Etcharry (dir.), La Grande Guerre en musique. Vie et création musicales en France pendant la Première Guerre mondiale, Berne, Peter Lang.

Doire, René (1916), «À nos lecteurs », Le Courrier musical, vol. 18, [s. no.] (1 ${ }^{\text {er }}$ décembre), p. 1.

Doire, René (1917), "Wagner ou le péril musical ? », Le Courrier Musical, vol. 19, [s. no.] (juillet-août), p. 267-269.

Duchesneau, Michel (1996), «La musique française pendant la guerre (1914-1918). Autour de la tentative de fusion de la Société nationale de musique et de la Société musicale indépendante ", Revue de Musicologie, vol. 82, nº 1, p. 123-153.

Fauré, Gabriel (1917), «Appel aux musiciens français », Le Courrier musical, vol. 19, [s. no.] (15 mars), p. 133.

Fulcher, Jane F., (2005), The Composer as Intellectual. Music and Ideology in France, 1914-1940, New York, Oxford University Press.

Gatti, Guido (1917), «L'étranger. Lettre d'Italie », Le Courrier musical, vol. 19, [s. no.] (1 $1^{\text {er }}$ février), p. 69.

Giesbrecht, Sabine (2014), Musik und Propaganda/Der Erste Weltkrieg im Spiegel deutscher Bildpostkarten, Epos Music, Osnabrück, Electronic Publising Universität Osnabruck.

Horne, John (2002), « Démobilisations culturelles après la Grande Guerre », 14-18, Aujourd'hui, Today, Heute, $\mathrm{n}^{\circ} 2$ (mai), p. 45-53.

Huré, Jean (1919a), «Du rythme dans la musique médiévale », Le Courrier musical, vol. 21, nº 4 (aoûtseptembre), p. 212-213.

Huré, Jean (1919b), "La fugue, composition libre, et la fugue d'école », Le Courrier musical, vol. 21, $\mathrm{n}^{\mathrm{o}} 15$ (1 $1^{\mathrm{er}}$ octobre), p. 230-231.

Huybrechts, Dominique (1999), 1914-1918. Les musiciens dans la tourmente : compositeurs et instrumentistes face à la Grande Guerre, Anserœul, Scaldis.

Jeismann, Michael(1992), Das Vaterland der Feinde. Studienzumnationalen Feindbegriff und Selbstverständnis in Deutschland und Frankreich (1792-1918), Stuttgart, Klett-Cotta. 
Kelly, Barbara (2013), Music and Ultramodernism in France. A Fragile Consensus, 1913-1939, Woodbridge, Boydell Press.

Knosp, Gaston (1905), "La presse musicale en Allemagne », Le Courrier musical, vol. 8, $\mathrm{n}^{\circ} 20$ (15 octobre), p. 570-574.

Koechlin, Charles (1917), «Esthétique ? (réponse à V. d'Indy) ", Le Courrier musical, vol. 19, [s. no.] (15 février), p 79-80.

Koechlin, Charles (1918), «La vie musicale pendant la guerre », Gazette des beaux-arts, [s. no.] (janviermars), p. 107.

Laloy, Louis (1921), "Art Lyrique et budget », Le Courrier musical, vol. 23, nº 6 (15 mars), p. 89.

Leterrier, Sophie-Anne (2000), «Culture de guerre et musique nationale. La musique française dans la Grande Guerre ", dans Annette Becker et al., Chefs-d'euvre et circonstances, Arras, Archives départementales du Pas-de-Calais, p. 15-38.

Marin, Séverine (2012), " Le commis voyageur allemand. Une image mythifiée dans la France de 1900 », Entreprises et histoire, n ${ }^{\circ}$ 66, p. 177-193, https://www.cairn.info/revue-entreprises-et-histoire2012-1-p-177.htm, consulté le 3 juin 2016.

Mauclair, Camille (1911), "Analogies », Le Courrier musical, vol. 14, nº 4 (15 février), p. 118-121.

Mauclair, Camille (1919), "Wagner après la guerre », Le Courrier musical, vol. 21, n 1 (1 $1^{\text {er }}$ janvier), p. 3.

Montant, Jean-Claude (1988), « La propagande extérieure de la France pendant la Première Guerre mondiale », thèse de doctorat, Université Panthéon-Sorbonne.

Moore, Rachel (2014), " "À ne pas ouvrir pendant la guerre". L'Union sacrée et la mobilisation de l'édition musicale ", dans Florence Doé de Maindreville et Stéphan Etcharry (dir.), La Grande Guerre en musique. Vie et création musicales en France pendant la Première Guerre mondiale, Berne, Peter Lang, p. 253-270.

Nerra, Foulques (1919), «L'étranger. Lettre d'Angleterre », Le Courrier musical, vol. 21, no 10 (1 ${ }^{\text {er }}$ mai), p. $140-141$.

Nin, Joaquin (1919), "Cadences dans tous les tons », », Le Courrier musical, vol. 21, n 19 (15 novembre), p. 293-294.

Poueigh, Jean (1916), «La musique depuis la guerre », Le Courrier musical, vol. 17, [s. no.] (1 ${ }^{\text {er }}$ décembre), p. 8.

Prochasson, Christophe, et Anne Rasmussen (1996), Au nom de la patrie. Les intellectuels et la Première Guerre mondiale (1910-1919), Paris, La Découverte, p. 203-221.

Puget, Théophile (1918), « Les subventions aux Grands-Concerts », Le Courrier musical, vol. 20, [s. no.] (15 janvier), p. 28-30.

Sala, Massimiliano (dir.) (2014), Music and Propaganda in the short Twentieth Century, Turnhout, Brepols.

Segond-Genovesi, Charlotte (2009), "De 1'“Union Sacrée” à la "Tribune des débats". La politique éditoriale de la Gazette des classes du Conservatoire (1915-1918) ", dans Myriam Chimènes et al. (dir.), La Grande Guerre des musiciens, Lyon, Symétrie, p. 175-190.

Simon, René (1921), « La Musique moderne espagnole », Le Courrier musical, vol. 23, n 2 (15 janvier), p. $22-23$.

Stany, Jean (1919), « La musique religieuse et son insuffisance. Causes et remèdes », Le Courrier musical, vol. $21, \mathrm{n}^{\circ} 18$ (15 novembre), p. 276-277.

Temkin, Moshik (2015), "Culture vs. Kultur, or a Clash of Civilisations. Public Intellectuals in the United States and the Great War ", The Historical Journal, vol. 58, n 1 (mars), p. 157-182, https://www.cambridge.org/core/journals/historical-journal/article/culture-vs-kultur-ora-clash-of-civilizations-public-intellectuals-in-the-united-states-and-the-great-war-19171918/ E3EA756634522624F57F2ACFAB2720C0, consulté le 15 avril 2017. 
Tenroc, Charles (1917a), «Le problème de l'édition française de musique », Le Courrier musical, vol. 19, [s. no.] (1 $1^{\text {er }}$ février $)$, p. 54-55.

Tenroc, Charles (1917b), "Quelques documents à propos du problème de l'édition française de musique », Le Courrier musical, vol. 19, [s. no.] (1 ${ }^{\mathrm{er}}$ avril), p. 164-167.

Tenroc, Charles (1917c), «Vers la libération de la facture française », Le Courrier musical, vol. 19, [s. no.] ( ${ }^{\text {er }}$ décembre), p. 385-390.

Tenroc, Charles (1918a), « Le nationalisme musical », Le Courrier musical, vol. 20, [s. no.] (septembreoctobre), p. 273-279.

Tenroc, Charles (1918b), "Une école franco-américaine », Le Courrier musical, vol. 20, [s. no.] ( $1^{\text {er }}$ décembre), p. 362.

Tenroc, Charles (1919a), «Comment il faut aimer la Muse », Le Courrier musical, vol. 21, $\mathrm{n}^{\circ} 7$ (1 $1^{\mathrm{er}}$ avril), p. 97.

Tenroc, Charles (1919b), "Comment il faut aimer la Muse (suite) », Le Courrier musical, vol. 21, nº 8 (15 avril), p. 115-116.

Tenroc, Charles (1921), «Les théâtres. Académie Nationale : La Walkyrie », Le Courrier musical, vol. 23, $\mathrm{n}^{\circ} 2$ (15 janvier), p. 24.

Vallas, Léon (1919), «Après la victoire », Le Courrier musical, vol. 21, nº 6 (15 mars), p. 83.

Villa, Georges (1919a), «Camille Chevillard préparant une attaque... », Le Courrier musical, vol. 21, $\mathrm{n}^{\circ} 5$ (1 ${ }^{\text {er }}$ mars), [n. p.].

Villa, Georges (1919b), "Anniversaire. Enfin Berlioz surgit, triomphant, sur les bords du Rhin... », Le Courrier musical, vol. 21, nº 6 (15 mars), [n. p.].

Vuillemin, Louis [pseud. Monsieur Soupir] (1919), "Variations sans thème. À MM. les éditeurs de musique ", Le Courrier musical, vol. 21, nº 4 (15 février), p. 61.

Vuillemin, Louis (1923), « Notes sans mesure. Concerts métèques ", Le Courrier musical, vol. 25, n 1 (1 ${ }^{\text {er janvier}), ~ p . ~} 4$.

Vuillermoz, Émile (1916), Lettre à Charles Koechlin, 23 août, inédite, Archives Charles Koechlin.

Watkins, Glenn (2003), Proof Through the Night. Music and the Great War, Berkeley, University of California Press. 